\title{
Traduire
}

Revue française de la traduction

234 | 2016

La traduction, un sport de haut niveau

\section{Traduction sous adrénaline}

\section{Heather Watson}

Traducteur : Hélène Ladjadj

\section{(2) OpenEdition}

\section{Journals}

\section{Édition électronique}

URL : http://journals.openedition.org/traduire/802

DOI : $10.4000 /$ traduire.802

ISSN : 2272-9992

Éditeur

Société française des traducteurs

Édition imprimée

Date de publication : 15 juin 2016

Pagination : 55-59

ISSN : 0395-773X

Référence électronique

Heather Watson, «Traduction sous adrénaline», Traduire [En ligne], 234 | 2016, mis en ligne le 15 juin 2018, consulté le 07 mai 2019. URL : http://journals.openedition.org/traduire/802 ; DOI : 10.4000/ traduire.802 


\section{Traduction sous adrénaline}

\section{Heather Watson}

\section{Au départ}

Mes premiers pas dans le domaine de la course moto datent de 2006, avec le Championnat du monde de vitesse moto puis, à partir de 2008, le Championnat du monde de Superbike. Si ma fonction officielle a toujours été " chargée de presse ", sa réalité, impliquant des rencontres avec des équipes italiennes et des concurrents internationaux dans le cadre des courses, exigeait bien sûr des aptitudes diverses et notamment la traduction et l'interprétation.

Petit flashback... Titulaire d'un BA (diplôme de premier cycle d'une université britannique, 2001) en italien et en espagnol, j'ai principalement travaillé en Italie depuis, hormis une courte période passée à Londres. J'ai commencé chez Ducati Motor Holding en 2004 à Bologne, où j'étais chargée de la relecture et de la mise à jour du site internet avant de décrocher, en 2008, le poste dont je rêvais depuis un bon moment : travailler pour le fameux département Corse (Courses) au titre de chargée de presse de l'équipe officielle Ducati au Championnat du monde de Superbike. Cette compétition internationale, consacrée aux motos dérivées des modèles de série, se déroule tous les ans de février à octobre, avec des courses sur circuit en Australie, au Qatar, en Malaisie, aux États-Unis, en Thaillande et dans divers pays européens. Autrement dit, j'allais voyager partout dans le monde au fil du calendrier du championnat, pour m'occuper de la communication presse de l'équipe, de ses relations publiques, du programme des pilotes, des interviews, etc. Et si a priorije n'avais pas perçu l'aspect linguistique de ce rôle, j'ai vite compris que je n'aurais jamais pu m'en sortir sans ces compétences particulières. $\mathrm{Ma}$ pratique du métier de traductrice indépendante depuis une dizaine d'années - parallèlement à mes activités de RP - m'a certainement été très utile (tout comme mon travail de RP m'a aidée à acquérir des compétences utiles pour mon activité de traduction).

Les équipes, en majorité italiennes, avec lesquelles j'ai travaillé ces huit dernières saisons sont établies en Italie et composées d'Italiens, hormis les pilotes qui peuvent venir de partout dans le monde. J'ai ainsi travaillé avec des champions originaires d'Australie, du Royaume-Uni, 
d'Espagne, du Japon, d'Italie et de France. Sur les circuits, bien sûr, le rythme est effréné. En outre, les intervenants, des mécaniciens aux partenaires techniques, des sponsors aux journalistes, viennent de différents pays et parlent donc des langues très diverses.

\section{En Italie}

L'une des activités essentielles à la fin de chaque journée sur le circuit est la rédaction des communiqués de presse nécessaires pour informer les médias internationaux des performances de l'équipe et des avis des pilotes, positifs ou négatifs. Chaque équipe, selon sa nationalité et celles de ses pilotes, fournit des communiqués en différentes langues selon ses besoins, l'anglais restant bien sûr omniprésent. Pour ma part, j'ai toujours eu à écrire en anglais et en italien.

Curieusement j'ai tendance à aborder le problème "à l'envers ", c'est-à-dire que j'écris d'abord en italien (ma langue B) et après seulement en anglais. J'ai vite compris que cette solution était la plus efficace et la plus rapide car, rédigé directement selon la syntaxe italienne, le texte " coule " naturellement, alors qu'il me serait bien plus compliqué de rendre en italien des phrases composées dans ma langue maternelle.

L'élément clé, c'est le temps - ou plutôt son absence ! II faut écrire, corriger et envoyer les communiqués le plus vite possible après les épreuves du jour pour publication par les journalistes, en ligne sur un site web ou dans les journaux du lendemain matin. Les principaux journalistes spécialisés étant à nos côtés sur les circuits, je dois souvent anticiper les propos d'un pilote pour respecter des délais de publication très serrés. Le niveau de stress est donc décuplé, car ces communiqués doivent être bien écrits, précis et prêts en 30 minutes chrono - il n'y a donc aucune marge d'erreur possible. II faut être clair, sans ambiguité, afin d'éviter toute erreur d'interprétation ou déformation des faits ou des propos d'un pilote par la presse. C'est pourquoi un membre de l'équipe relit toujours mes textes avant leur envoi.

La traduction intervient aussi pour la communication via les réseaux sociaux. Les équipes alimentent plusieurs fils sur Facebook, Twitter et Instagram, qui doivent tous être mis à jour au quotidien, leurs contenus traduits bien entendu. La brièveté requise sur Twitter implique que les tweets en anglais doivent souvent être adaptés, et pas simplement traduits, pour la version italienne. J'ai certainement fait quelques progrès en ce qui concerne la concision !

Au cours d'une saison, les documents à traduire ne manquent pas, des dossiers de presse aux outils de marketing, des discours jusqu'aux courriels échangés entre collègues.

\section{Au-delà de la traduction}

Ce travail suppose aussi une interaction, plus délicate qu'on pourrait le penser, avec les médias. En effet, si la plupart des journalistes présents sur les circuits maîtrisent l'anglais, pour les 
autres une connaissance même rudimentaire d'autres langues (espagnol, français et allemand en ce qui me concerne) peut se révéler précieuse ! Comme c'est sans doute le cas dans d'autres secteurs médiatiques, certains journalistes spécialisés peuvent se montrer, disons, un peu difficiles à gérer. lls pourraient ainsi chercher à vous piéger, vous ou le pilote, avec une question tendancieuse, ou à déformer vos propos à la moindre occasion! II faut donc être vigilant lorsque vous vous exprimez, surtout dans une langue qui n'est pas la vôtre, ou lorsque vous traduisez les propos d'un pilote.

Si la plupart des pilotes avec lesquels j'ai travaillé parlent parfaitement anglais, il reste quelques exceptions et même s'ils parlent anglais, il est peu probable qu'ils maîtrisent aussi l'italien. Les interviews se passant généralement dans l'une de ces langues, je suis régulièrement sollicitée pour l'interprétation de l'anglais vers l'italien et vice versa. N'étant pas interprète professionnelle, j'ai toujours le trac, mais il s'agit en général d'un journaliste que nous connaissons bien ou de fans simplement ravis de rencontrer leur pilote préféré. Le cadre n'est donc pas très formel.

La course moto possède bien sûr sa terminologie technique et il convient d'en connaître les termes et expressions, notamment lorsqu'il faut interpréter pour la presse, compte tenu de la typologie des questions. Clarté et précision s'imposent, surtout lors d'un enregistrement pour la télévision.

Dans ce cas, tout dépendra des préférences de la production concernant la diffusion - en direct ou en différé. Souvent, l'équipe de tournage optera pour une simple conversation à deux sans interprète de liaison; s'il s'agit d'un différé, j'interviens pour que l'équipe de tournage obtienne ce dont elle a besoin auprès du pilote, mais ma contribution est retravaillée, parfois remplacée par des sous-titres au besoin, pour un résultat " propre ". En revanche, si la production a besoin d'entendre la voix de la personne interrogée, le pilote peut être préparé pour s'exprimer dans une langue qu'il ne connaît pas assez, ou bien on me demande d'intervenir et ses propos sont suivis de mon interprétation.

Souvent les pilotes, pas très au fait de ce genre de problèmes, ont tendance à répondre aux questions de manière décousue. Comme je pratique une interprétation consécutive plutôt que simultanée (les interviews étant généralement enregistrées, cela évite la confusion due au chevauchement des voix), je leur demande de ménager des pauses entre les phrases pour me permettre d'interpréter leurs propos.

Autre domaine dans lequel je n'avais aucune expérience, celui du sous-titrage. Les équipes préparant tous les ans le lancement et la présentation des événements, on m'a parfois demandé de sous-titrer en anglais les vidéos italiennes. Malgré mon inexpérience, j'ai vraiment beaucoup apprécié cet exercice car, au-delà de la transmission du sens, j'ai adoré le défi de la concision ou des périphrases pour respecter les restrictions de longueur. Je ne le fais pas souvent mais j'aimerais bien améliorer mes compétences dans ce domaine. 


\section{Forcella/forcellone}

Bien entendu, j'ai emmagasiné une myriade de termes techniques, entendus si souvent en italien au fil des ans que j'ai parfois l'impression de mieux les connaître dans ma deuxième langue! (Je me demande souvent si je suis la seule dans ce cas ou si c'est la même chose pour tous ceux qui séjournent longtemps dans un autre pays...)

Même un pilote anglophone, qui ne connaîtrait pas beaucoup d'italien, pourrait très bien maîtriser presque tous les mots désignant les différents éléments de la moto - on apprend très vite le vocabulaire technique lorsque l'on est " en immersion "!

Pour traduire, surtout dans cet environnement tendu, soumis à l'urgence, je dois être très attentive aux termes spécialisés. Certains prêtent aisément à confusion - forcella et forcellone par exemple (fourche et bras oscillant, respectivement), ou ciclistica, renvoyant ici au réglage de la suspension et pas du tout au vélo. Giri (tours) peut désigner les tours de circuit ou le régime du moteur (outre tous les autres sens sans lien avec la moto). Prove signifie généralement essais, mais c'est aussi le mot désignant les séances d'entraînement pendant le weekend de course. Autre terme prisé dans le sport moto, le mot anglais feeling, très utilisé en italien, bizarrement à mon avis, pour décrire la relation du pilote avec sa moto, dans des phrases que je rendrais en anglais par " Je me sens désormais en symbiose avec ma moto".

\section{Un travail, plusieurs facettes...}

Rien d'étonnant si le motocyclisme et sa version sportive sont devenus mes spécialités et si une grande part de mon travail pour des agences de traduction concerne ce domaine.

Ma longue expérience m'a valu le privilège d'être sollicitée pour traduire un livre de l'italien en anglais, il y a deux ans environ. II s'agissait d'un hommage au pilote motocycliste Marco Simoncelli, victime d'un tragique accident lors d'une course de MotoGP en 2011. Le texte était bien sûr truffé de vocabulaire spécialisé, mais tout à fait dans mes cordes. J'ai d'autant plus apprécié de travailler sur ce livre que j'avais eu l'occasion de rencontrer Marco plusieurs fois - ce qui a ajouté à cette mission une forte charge émotionnelle.

(Un membre éminent de la presse britannique m'a dit un jour que je m'étais très bien sortie de cette traduction, d'autant qu'il venait de lire un autre livre consacré à la moto dans lequel le mot " roue " a été traduit systématiquement par " cerceau ". Le mot italien cerchio peut en effet désigner un cerceau et une roue, mais un traducteur digne de ce nom sait que, s'agissant d'une moto, on parle de roues, il me semble ?!)

Le vrai défi auquel j'ai été confrontée avec ce livre est venu de l'inclusion ponctuelle de mots et expressions en romagnol, dialecte italien de la Romagne, parlé par Simoncelli, sa famille et 
nombre de ses amis. II m'a donc fallu faire des recherches assez poussées pour comprendre exactement le sens et le rendre avec précision en anglais. J'ai aussi rencontré ce problème sur les circuits, les Italiens possédant une vaste diversité de langues régionales. II m'est arrivé de demander à un pilote romain de m'éclairer lorsque je n'avais pas saisi la " note locale " dont il avait agrémenté ses propos et une fois j'ai entendu une interview avec un pilote - originaire de Bari - dont l'italien a semblé si étrange à mes oreilles que j'ai pensé qu'il s'agissait d'un non-Italien faisant des efforts linguistiques !

\section{Pour finir}

Trêve de plaisanterie, je conclurai en disant qu'il s'agit d'une tâche exigeante mais certainement facilitée par mon bilinguisme. Si ce travail peut éventuellement être accompli sans posséder une deuxième langue (les chargés de presse monolingues ont recours à un traducteur), cette maîtrise des langues facilite grandement ma vie et celle de mes employeurs (!). En outre, j'ai pu améliorer et étendre mes compétences au-delà de la simple traduction - interprétation, sous-titrage et montage, notamment. Traduction et course de motos : deux mondes - les miens - au rythme effréné, intense, qui ont bien plus en commun qu'on ne l'imagine.

info@racinglines.org

Traduit de l'anglais par Hélène Ladjadj.

Linguiste convaincue depuis qu'elle a découvert le français et le latin à l'âge de neuf ans, Heather Watson est traductrice et rédactrice depuis une dizaine d'années. Britannique, elle vit aujourd'hui en ÉmilieRomagne (Italie) où elle traduit et relit de l'italien, et parfois de l'espagnol et du français, vers l'anglais. Ses domaines de spécialisation sont notamment le secteur automobile, la communication et le marketing, le tourisme, les arts, la presse, les produits alimentaires et les boissons, ainsi que le luxe. Membre du Chartered Institute of Linguists, elle est titulaire d'un diplôme de premier cycle en langues modernes et d'un Master en traduction. Elle consacre également son activité de journaliste à temps partiel aux sports mécaniques, un univers qui est devenu le sien depuis de nombreuses années.

Site web : www.racinglines.org 